\title{
An Overview of Thermal Distortion Modeling, Analysis, and Model Validation for the JWST ISIM Structure
}

\author{
John D. Johnston ${ }^{1}$ \\ NASA Goddard Space Flight Center, Greenbelt, MD, 20771 \\ Emmanuel Cofie ${ }^{2}$ \\ MEGA Engineering, Silver Spring, MD, 20901
}

\begin{abstract}
This paper describes thermal distortion modeling, analysis, and model validation activities completed in support of the design and qualification of the JWST ISIM Structure. An overview of the building blocks approach utilized to develop the structural design and modeling capabilities to meet thermal distortion performance requirements will be provided. This effort culminated in a cryogenic (35 K) distortion test of the flight ISIM Structure hardware, the Cryoset Test that both verified the thermal distortion performance and provided data for validation of the structural models. An overview of the modeling, analysis, and model validation development effort will be discussed, and the results of the Cryoset Test and the subsequent model validation will be described.
\end{abstract}

\section{Introduction}

$\mathrm{T}$ HE James Webb Space Telescope (JWST) is a large, infrared-optimized space telescope scheduled for launch in 2014. The JWST consists of an optical telescope element (OTE), an integrated science instrument module (ISIM), a spacecraft, and a sunshield (Figure 1, References 1-2). The JWST Integrated Science Instrument Module (ISIM) consists of the JWST science instruments (NIRCam, MIRI, NIRSpec), a fine guidance sensor (FGS), the ISIM Structure, and thermal and electrical subsystems (Figure 2). The ISIM Structure is a large (approximately $2 \mathrm{~m}$ $\times 2 \mathrm{~m} \times 1.5 \mathrm{~m}$ ), bonded composite frame that serves as the metering structure between the instruments/guider and the telescope (References 34). JWST's instruments will be designed to work primarily in the infrared range of the electromagnetic spectrum, and the instruments and telescope operate at cryogenic temperatures $(\sim 35 \mathrm{~K}$ for the instruments). Thermal distortion performance is critical to maintaining the alignment of the instruments to the ISIM Structure, and significant effort has been expended on the development of capabilities to predict and measure cryogenic thermal distortion. This paper will discuss thermal distortion modeling, analysis, and model validation activities completed in support of the design and qualification of the ISIM Structure. Topics will include: ISIM Structure optomechanical performance requirements, and model validation completed in support of thermal distortion analysis of the ISIM Structure. Particular emphasis will be placed on discussion of recently completed thermal distortion model validation activities for the ISIM Structure

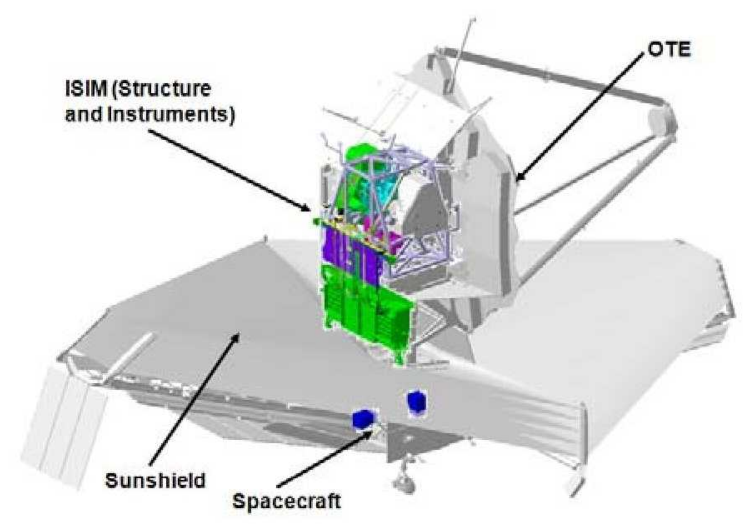
involving a cryogenic $(35 \mathrm{~K})$ distortion test of the flight ISIM Structure hardware that both verified the distortion performance and provided data for validation of the structural models.

${ }^{1}$ Aerospace Engineer, Mechanical Systems Analysis and Simulation Branch, Code 542, NASA Goddard Space Flight Center, Greenbelt, MD 20771.

${ }^{2}$ Structural Engineer, Mega Engineering, 10701 Harper Avenue, Silver Spring, MD 20901. 


\section{ISIM Structure Development}

The ISIM Structure development followed a building blocks approach starting with the development and testing of material samples and culminating with the structural verification program for the protoflight hardware. The first step in the development was material selection and characterization. During this stage the composite laminates utilized in the construction of the ISIM Structure were selected. Extensive coupon testing to develop a materials database was completed, in particular to measure the CTE between room temperature and cryogenic cold survival temperature for all materials. During the second stage of the development program, bonded joints were designed, manufactured, and tested to assess performance and provide anchor points for both strength and distortion modeling. During the next

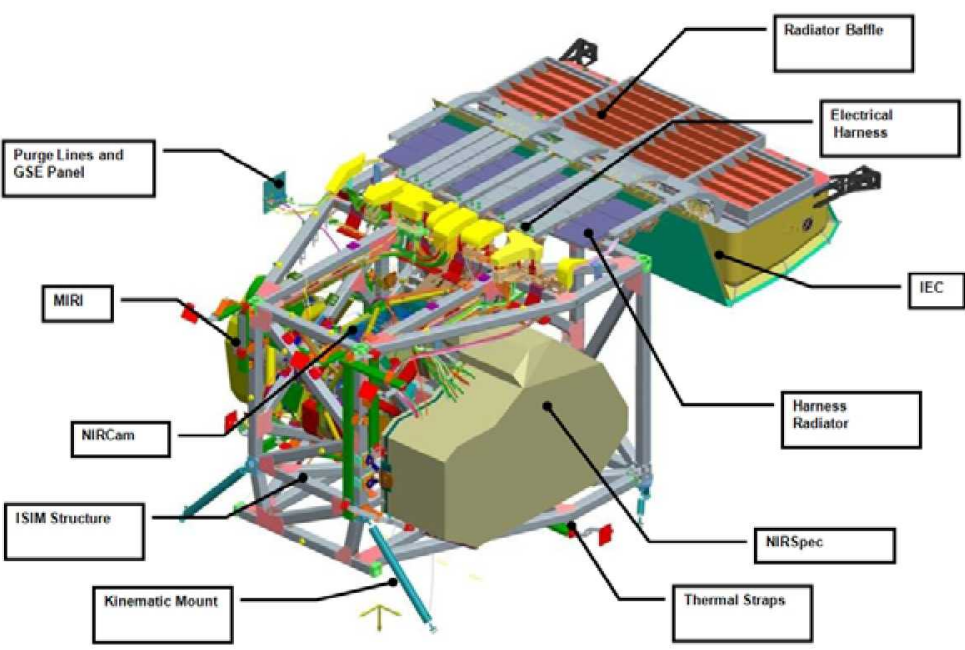

Figure 2. Integrated Science Instrument Module (ISIM). stage a 3D frame representative of a partial instrument bay from the ISIM structure, the "breadbox", was designed manufactured, and subjected to thermal distortion testing down to cryogenic operating temperatures primarily for the purpose of thermal distortion model validation. Finally, the flight ISIM Structure was designed, manufactured, and is currently in the process of completing its structural verification program. The first of the major environmental tests, the Cryoset Test, was completed in May 2010 (Figure 3). Following this test will be a Cryoproof test, a modal survey, and strength testing via a centrifuge test and static pull testing. During this test the ISIM Structure was cycled between ambient and cryogenic temperatures and metrologized using a photogrammetry system. During the Cryoset Test it was demonstrated that the structure meets its optomechanical performance requirements. Photogrammetry data was used to validation? the thermal distortion model predictions for cooldown performance between room temperature and cryogenic operating temperature.

\section{Thermal Distortion Modeling, Analysis, and Model Validation}

The ISIM Structure thermal distortion models are NASTRAN structural models of high fidelity $(>2$ million DOF). The structure is modeled using solid elements to capture fine details such as bond lines and bond shapes. A key aspect of the models is their linkage to a materials database populated by data specifically generated for the program. The model utilizes temperature-dependent CTE and stiffness properties to accurately prediction thermal distortion behavior in terms of both cooldown from ambient to cryogenic temperatures and stability at operational cryogenic temperatures.

The thermal distortion modeling and analysis development effort consisted of three main phases: (1) mesh convergence studies and analysis approach development, (2) preliminary model validation via development testing, and (3) final flight modeling and validation.

The mesh convergence studies were used to establish the mesh size for the global thermal distortion model. During this effort, high fidelity models of representative bonded joints served as the reference standard against which successive thermal distortion model mesh sizes could be compared. Ultimately, a balance between model accuracy and model size defined the final mesh sizes. There are two analysis approaches used in ISIM Structure thermal distortion modeling and analysis. The first is the nominal model, and the second is a stochastic model. The stochastic model is used to predict the model uncertainty due to factors such as material property variability. The stochastic model provides a mean prediction and an uncertainty band determined by multiplying the $95 \%$ confidence interval by a modeling uncertainty factor (MUF). As per project guidelines, modeling uncertainty factors are used to provide conservatism and margin in thermal distortion predictions. Nominal model predictions are multiplied by a nominal MUF of 1.6, while stochastic model predictions use of MUF of 1.4.

Preliminary model validation was accomplished as part of the development effort for ISIM Structure composite bonded joint development. A series of three basic joint types (plug, $T$, and saddle) were designed, manufactured, 
tested for strength and thermal distortion as part of this effort. Thermal distortion models of the test articles were generated using the final mesh sizing from the mesh refinement study, and test results were used to validate the predictions from those models. A 3-D frame structure, the "breadbox", representative of a bay from the ISIM Structure and consisting of all the basic joint types was also designed, manufactured, and tested using the lessons learned and approaches developed during the joint level testing. A thermal distortion model of the "breadbox" was generated again using the mesh size parameters previously developed, and results from thermal distortion testing were used to validate the model. Figure 3 presents thermal distortion model validation results for the "breadbox" test article. Results are provided for nominal model predictions, stochastic model predictions, and test measurements for cooldown distortion from room temperature to $40 \mathrm{~K}$. The model validation criteria are that the nominal model predictions bound the measurements, and the stochastic model predictions envelop the test measurements. These results demonstrate that the model accurately predicts the cooldown distortion characterized during testing.
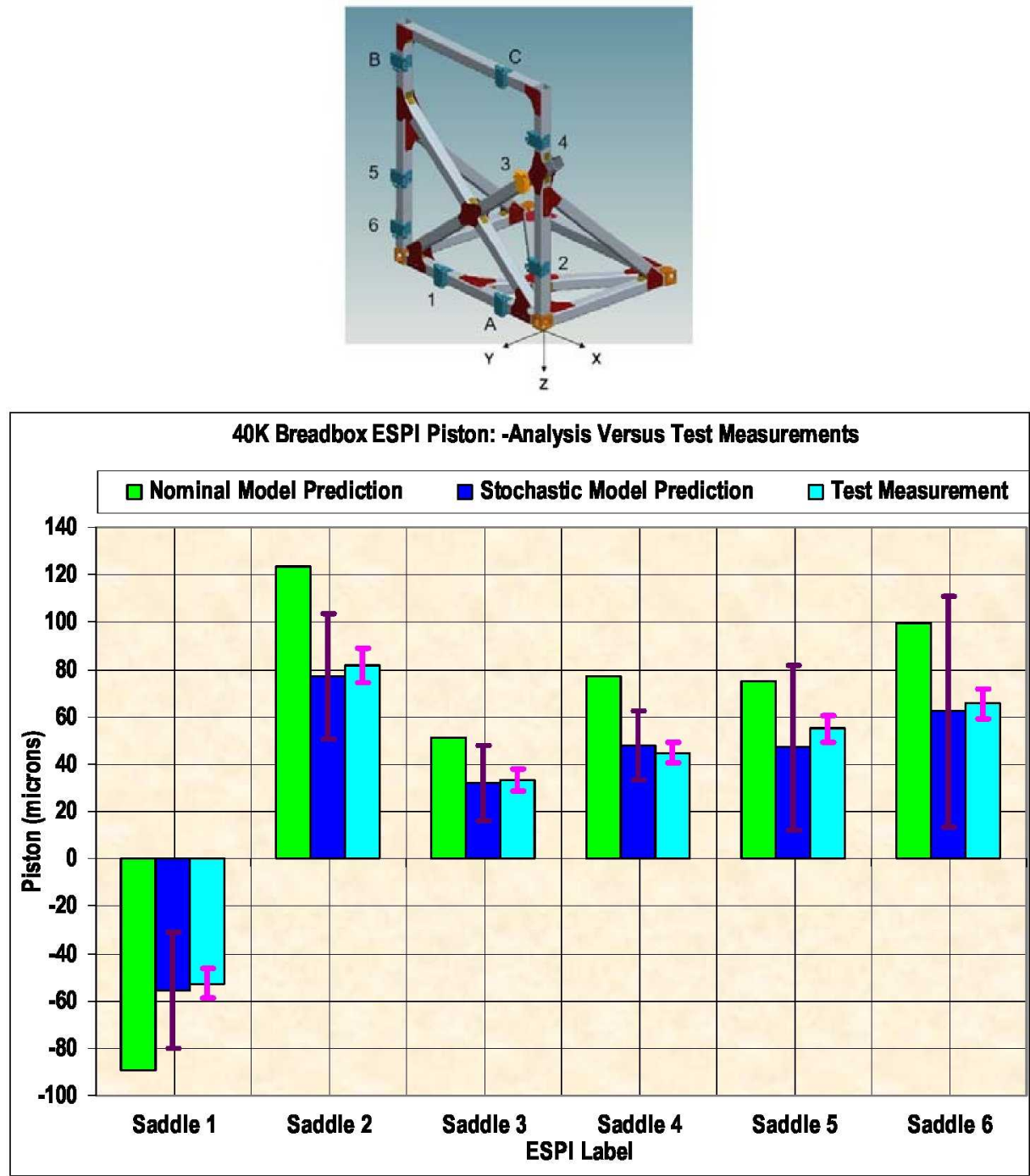

Figure 3. "Breadbox" Test Article Thermal Distortion Test Article and Model Validation Results for Cooldown Distortion.

Final model validation was accomplished by comparison of results from the ISIM Structure Cryoset Test with predictions from the thermal distortion model. A detailed model of the test setup, including both the flight hardware and the mechanical ground support equipment (MGSE), was generated to predict thermal distortion performance (Figure 4). The ISIM Structure model incorporated into the test setup model is the flight structure model with the addition of metrology tooling and targets used during the testing. Temperature sensor measurements taken during the test were used to generate temperature maps for the flight hardware and MGSE following the test. 
A custom photogrammetry system was developed specifically to metrologize the ISIM Structure during the Cryoset Test. This system provided sufficient accuracy (33 microns for translations, 0.5 arcmin for rotations) to

Figure 4. Cryoset Test Structural Model

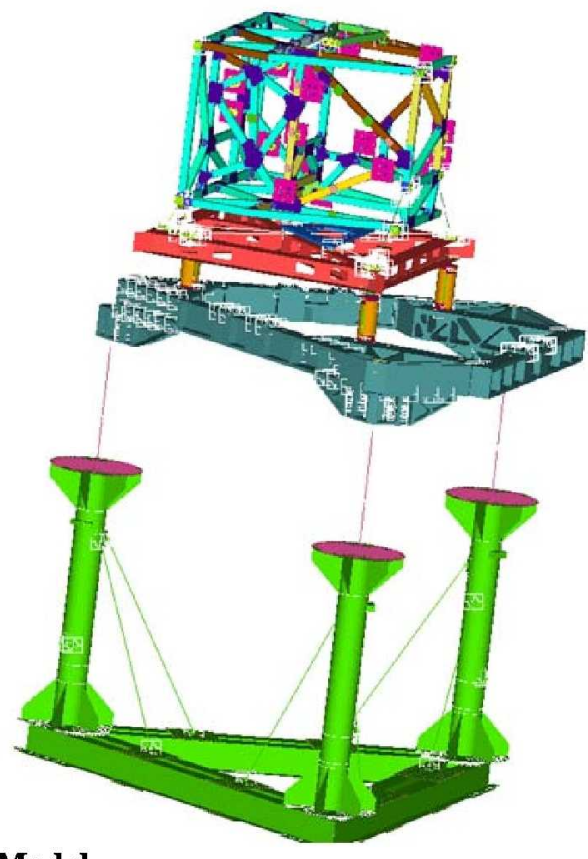

verify that ISIM Structure optomechanical performance requirements are satisfied and to provide data for validation of thermal distortion model predictions. Figure 5 provides a model validation example based on Cryoset Test results. The performance metric of interest in this example is distortion of the science instrument interfaces that are represented by Science Instrument Extender Plates (SEPs) during the test. The SEPs provide an array of photogrammetry targets to enable characterization of both translations and rotations. The results demonstrate that the nominal model predictions (FEA with $M U F=1.6$ ) bound the test measurements, and the stochastic model predictions (FEA) envelop or partially envelop the test measurements.

\section{Summary}

The full paper will provide additional details on the modeling and analysis approaches for ISIM Structure

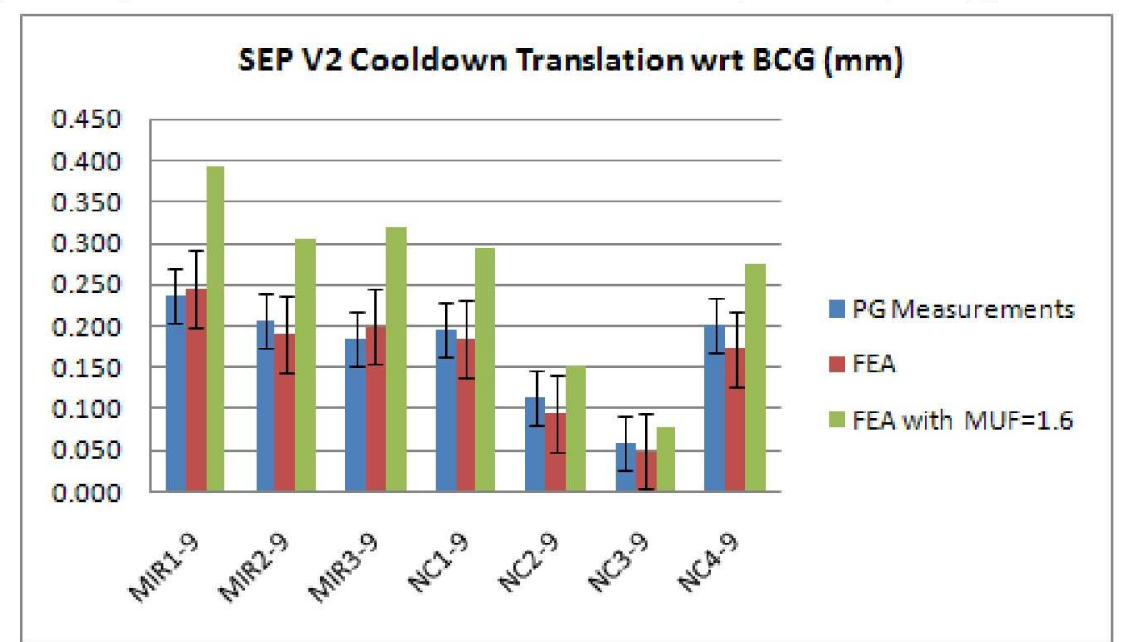

Figure 5. Model Validation Example from Cryoset Test 
thermal distortion. An overview of the various thermal distortion test setups and results will also be provided. Finally, detailed results regarding model validation will be provided for basic joints, the breadbox, and the flight ISIM Structure.

\section{Acknowledgments}

The authors would like to acknowledge the contributions of the following. The entire ISIM Structure team, in particular Eric Johnson, Jim Pontius, Jason Hylan, Doug McGuffy, Patrick Willaims, and Steve Breuder. Contributors to the thermal distortion analysis effort, in particular Paul Bagdanove and Steve Mariconti. Contributors to the test and metrology effort, including Ray Ohl, Maria Nowak, Joe Stock , Allan Crane, Jimmy Marsh, Stu Glazer, Paul Cleveland, Brian Comber.

\section{References}

1. "An overview of the James Webb Space Telescope (JWST) project," Phillip A. Sabelhaus, Doug Campbell, Mark Clampin, John Decker, Matt Greenhouse, Alan Johns, Mike Menzel, Robert Smith, Pam Sullivan, Proc. SPIE Vol. 5899, p. 241-254, UV/Optical/IR Space Telescopes: Innovative Technologies and Concepts II, August 2005.

2. "The James Webb Space Telescope instrument suite layout: optical system engineering considerations for a large deployable space telescope” Brent J. Bos, Pamela S. Davila, Matthew Jurotich, Gurnie Hobbs, Paul A. Lightsey, James Contreras, Tony Whitman, Proc. SPIE Vol. 5487, p. 734-745, Optical, Infrared, and Millimeter Space Telescopes, October 2004.

3. "Development and sizing of the JWST Integrated Science Instrument Module (ISIM) metering structure," Cengiz O. Kunt, John Johnston, Andrew Bartoszyk, Steve Hendricks, Proc. SPIE Vol. 6273, Optomechanical Technologies for Astronomy, July 2006.

4. "Design/analysis of the JWST ISIM bonded joints for survivability at cryogenic temperatures," Andrew Bartoszyk, John Johnston, Charles Kaprielian, Jonathan Kuhn, Cengiz Kunt, Benjamin Rodini, Daniel Young, Proc. SPIE Vol. 5868, p. 171-180, Optical Materials and Structures Technologies II, August 2005. 